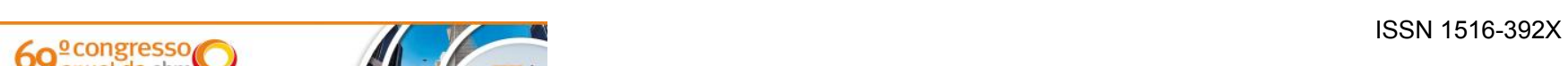

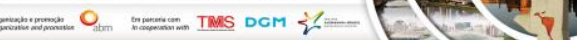

Tema: Mecânica da Fratura e Integridade Estrutural

\title{
POTENCIAL DE CORPOS DE PROVA CHARPY PRÉ-TRINCADOS (PCVN) NA DETERMINAÇÃO DE CURVAS R E DE PROPAGAÇÃO DE TRINCA À FADIGA DE AÇOS ESTRUTURAIS*
}

\author{
Gustavo Henrique Bolognesi Donato ${ }^{1}$ \\ Rodrygo Figueiredo Moço ${ }^{2}$ \\ Tatiane Rossi Merlo² \\ Felipe Cavalheiro Moreira ${ }^{3}$ \\ Maurício de Carvalho Silva ${ }^{1}$
}

\section{Resumo}

Ensaios de curva $R$ ( $J$ vs. $\Delta a$ - crescimento dúctil de defeitos) e de propagação de trincas por fadiga (da/dN vs. $\Delta K)$ são balizados por normas como a ASTM E1820, ISO 12135 e E647, as quais exigem dimensões mínimas para as amostras $C(T)$ ou $S E(B)$ a fim de garantir condições SSY e portanto a validade de $K$ e $J$. Entretanto, tais exigências usualmente conduzem a dimensões expressivas que dificultam a retirada de amostras de chapas finas ou mesmo de muitas estruturas reais. Como alternativa, estudos de Koppenhoefer e Dodds (1998) e mais recentemente de Shin e Lin (2012) indicaram a possibilidade de uso de amostras em miniatura (inclusive Charpy contendo pré-trinca) para determinação de curvas $R$ e $d a / d N v s$. $\Delta K$. As grandes questões são a viabilidade de ensaio de amostras pequenas com repetibilidade e a adequada avaliação dos limites de validade das forças motrizes nestas geometrias, a fim de garantir condições de similitude. Como um passo nesta direção, este trabalho investiga o potencial de amostras Charpy pré-trincadas $(10 \times 10 \times 70 \mathrm{~mm})$ na determinação de curvas $R$ e $d a / d N$ vs. $\Delta K$. Inicialmente, ensaios de ambos os tipos foram realizados em amostras de um aço estrutural ferrítico empregando uma máquina universal MTS 810. Apesar das dimensões reduzidas dos espécimes, os ensaios se mostraram viáveis e apresentaram boa repetibilidade e estabilidade dos resultados experimentais. Ainda, boas práticas de ensaio puderam ser estabelecidas para suportar os trabalhos futuros com tais geometrias.

Palavras-chave: Espécimes reduzidos; Charpy pré-trincados; Curva $R$; Curva $d a / d N$ vs. $\Delta K$; Aços estruturais.

\section{POTENTIAL OF PRECRACKED CHARPY SPECIMENS (PCVN) FOR DETERMINING R CURVES AND FATIGUE CRACK GROWTH RATES OF STRUCTURAL STEELS \\ Abstract}

Mechanical testing regarding $R$ curves ( $J v s . \Delta a$ - ductile extension of cracks) and fatigue crack growth (da/dN vs. $\Delta K$ ) are supported by standards such as ASTM E1820, ISO 12135 and ASTM E647, which impose minimum dimensions to $C(T)$ and $S E(B)$ specimens in order to guarantee SSY conditions and therefore validity of $K$ and $J$. However, such requirements usually lead to large dimensions that make the extraction of samples from real structures sometimes unfeasible. As an alternative, results from Koppenhoefer and Dodds (1998) and more recently from Shin and Lin (2012) indicated the possibility of employing miniature specimens (including precracked Charpy geometries) for the experimental estimation of $R$ curves and $d a / d N v s . \Delta K$ evolutions. The key concerns are: i) the feasibility of performing the tests in such small specimens with repeatability; ii) to obtain valid crack driving forces during the tests, in order to guarantee similitude. As a step in this direction, this work examines the potential of precracked Charpy specimens $(10 \times 10 \times 70 \mathrm{~mm})$ for determining $R$ curves and $d a / d N v s . \Delta K$ evolutions. Both kinds of tests were conducted on samples made of a structural ferritic steel using a MTS 810 testing machine. Despite the reduced dimensions of the samples under investigation, all tests were successful and presented repeatability and stability on the obtained experimental results. Furthermore, best practices for testing PCVN specimens could be established to support future developments in the field.

Keywords: Reduced Samples; Precracked charpy; $R$ Curves; da/dN vs. $\Delta K$; Structural steels.

1 Engenheiro Mecânico, Doutor, Docente, Departamento de Engenharia Mecânica, Centro Universitário da FEl, São Bernardo do Campo, SP, Brasil; gdonato@fei.edu.br.

2 Aluno de Iniciação Científica, Departamento de Engenharia Mecânica, Centro Universitário da FEl, São Bernardo do Campo, SP, Brasil.

3 Aluno de Mestrado do Departamento de Engenharia Mecânica do Centro Universitário da FEl, São Bernardo do Campo, SP, Brasil.

\footnotetext{
* Contribuição técnica ao 69 Congresso Anual da ABM - Internacional e ao 14ํㅡㄹ ENEMET - Encontro Nacional de Estudantes de Engenharia Metalúrgica, de Materiais e de Minas, 21 a 25 de julho de 2014, São Paulo, SP, Brasil.
} 


\section{INTRODUÇÃO}

Ensaios de curva $R$ ( $J$ vs. $\Delta a$ - crescimento dúctil de defeitos) e de propagação de trincas por fadiga ( $d a / d N$ vs. $\Delta K$ ) fornecem propriedades mecânicas que representam a base para diversos protocolos de avaliação de integridade estrutural, aceitação de defeitos e previsão de vida de componentes de alta responsabilidade, especialmente em setores como aeronáutico, óleo e gás, nuclear e automotivo [1,2]. Usualmente, tais ensaios são balizados por normas como a ASTM E1820 [3], ISO 12135 [4] e E647 [5], as quais pelo caráter geral de aplicação exigem dimensões mínimas para as amostras $\mathrm{C}(\mathrm{T})$ ou $\mathrm{SE}(\mathrm{B})$ a fim de garantir condições SSY (escoamento de pequena monta - do inglês Small Scale Yielding) e portanto a validade de $K$ e/ou da integral $J$, respectivamente os principais parâmetros da Mecânica da Fratura Linear Elástica (MFLE) e Elasto-Plástica (MFEP). A busca pela validade das forças motrizes de trinca $K$ e/ou integral $J$ durante os ensaios objetiva garantir que tais parâmetros sejam descritores dos campos de tensões à frente da trinca (os quais governam, na zona de processo de fratura, os processos de propagação em fadiga e rasgamento dúctil). Isto é de suma importância para que as propriedades mecânicas de laboratório possam ser utilizadas em aplicações reais utilizando as considerações de transferabilidade da mecânica da fratura monoparamétrica [1]. Entretanto, tais exigências dimensionais usualmente conduzem a amostras de grandes dimensões que dificultam (senão inviabilizam) a retirada de amostras de chapas finas ou mesmo de muitas estruturas reais. Para fins de ilustração, amostras confeccionadas em aços estruturais ferríticos de uso nos dias atuais para sistemas dutoviários (p.ex.: aços API 5L X65 a X80 [6]) somente conduzem a forças motrizes válidas se contarem com espessuras acima de $\sim 3 / 4$ ou 1 polegada [3]. Tais espessuras incorrem em dimensões planares facilmente superiores a centenas de milímetros.

Como alternativa a tais amostras, diversos estudos indicaram a possibilidade de uso de amostras em miniatura (inclusive geometria Charpy contendo pré-trinca - PCVN) para determinação de curvas $R$ e $d a / d N$ vs. $\Delta K$. Bons exemplos de tais desenvolvimentos são os trabalhos de Koppenhoefer e Dodds [7,8], Chaouadi e Puzzolante [9], Puigh et al. [10], Sriharsha et al. [11], Li e Stubbins [12] e recentemente em 2012 Shin e Lin [13]. Embora de grande interesse, muitos destes trabalhos possuem poucos resultados experimentais e não endereçam com clareza as melhores práticas para testes de geometrias como as mencionadas. Adicionalmente, uma boa parte destas referências endereça considerações sobre amostras miniaturizadas, mas não trata especificamente da geometria PCVN .

As grandes questões neste caso são a viabilidade de ensaio de amostras pequenas com estabilidade e repetibilidade e a adequada avaliação dos limites de validade das forças motrizes nestas geometrias, a fim de garantir condições de similitude. Como um passo nesta direção, este trabalho investiga o potencial de amostras Charpy prétrincadas (PCVN - 10x10x70mm) na determinação de curvas $R$ e da/dN vs. $\Delta K$. Inicialmente, geometrias Charpy tiveram de ser adaptadas para ensaios de fratura e fadiga. Na sequência, ensaios de ambos os tipos foram realizados para um aço estrutural ferrítico ASTm A36 empregando uma máquina universal MTS 810, seguidos de catelosa análise fractográfica e pós-processamento dos resultados.

\subsection{Metodologia de Determinação de Curvas $J-R$}

As chamadas curvas $R$ quantificam a relação entre força motriz de trinca (no caso integral $J$ ) e extensão do defeito $(\Delta a)$ e são de grande relevância para quantificar a resistência ao fraturamento de materiais que exibem rasgamento dúctil. A técnica

\footnotetext{
* Contribuição técnica ao $69^{\circ}$ Congresso Anual da ABM - Internacional e ao 14ํㅡㄹ ENEMET - Encontro Nacional de Estudantes de Engenharia Metalúrgica, de Materiais e de Minas, 21 a 25 de julho de 2014, São Paulo, SP, Brasil.
} 


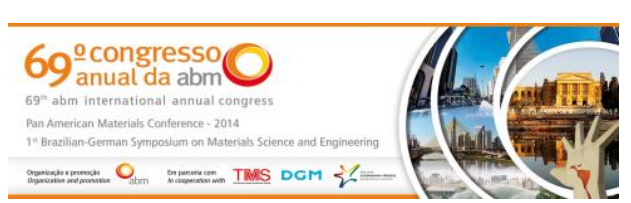

experimental mais aplicada para sua determinação é uma metodologia monoespécime [1,3] que segue o seguinte protocolo: i) inicialmente, a amostra é monotonicamente carregada e sofre diversos descarregamentos parciais durante a extensão da trinca (vide Figura 1a). Dados de carga $(P)$ e deslocamento da boca da trinca (CMOD - $V$ ) são aquisitados durante todo o teste; ii) a computação das áreas elásticas e plásticas para cada ponto da curva (respectivamente $A_{e l}$ e $A_{p l}$ ) permitem calcular a integral $J$ instantânea empregando o método eta $(\eta)[1,3]$; iii) cada descarregamento parcial, por sua vez, fornece informações sobre a flexibilidade instantânea da amostra (compliance $C$ - a qual é resultado do comprimento instantâneo da trinca). Normas correntes fornecem relações polinomiais que estimam a profundidade da trinca com base em $C$ (detalhes podem ser encontrados no trabalho de Donato e Moreira [14]). Considerando dois descarregamentos subsequentes " $i$ " e " $n$ " (Figura 1a), os respectivos pontos $J$ - $\Delta a$ são apresentados em detaque na Figura 1(b). Mais informações sobre região de validade da curva e significância para a avaliação de integridade estrutural estão disponíveis em $[1,3]$.

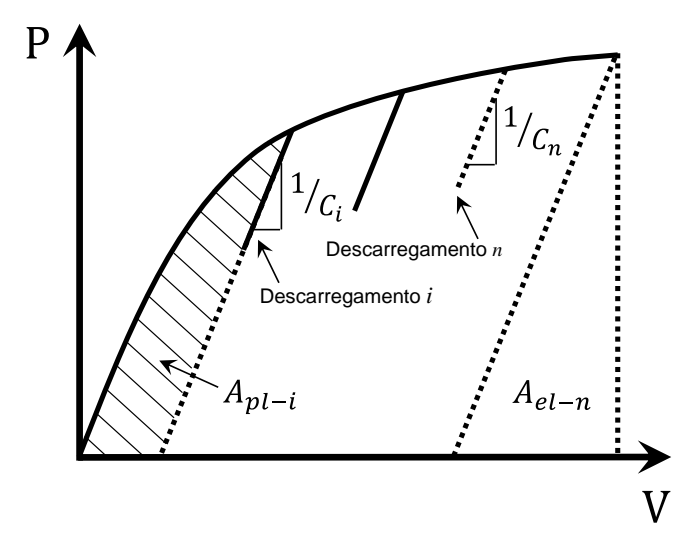

(a)

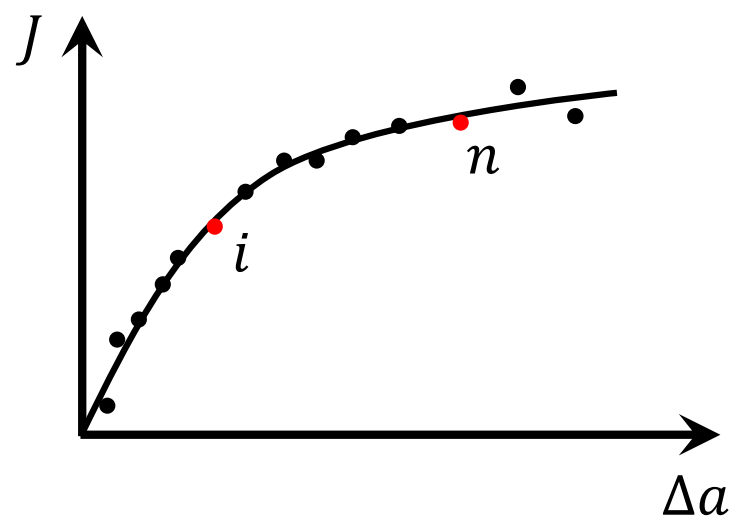

(b)

Figura 1. (a) Curva de carga $(P)$ vs. deslocamento $(C M O D-V)$ incluindo descarregamentos parciais e flexibilidades elásticas $C$. (b) Curva $J-R$ esquemática relacionando $J$ aplicado à extensão de trinca $\triangle a$.

\subsection{Metodologia de Determinação de Curvas $d a / d N$ vs. $\Delta K$}

Ensaios de propagação de trinca em fadiga também empregam amostras de mecânica da fratura (na maioria dos casos $\mathrm{C}(\mathrm{T})$ ) e todos os detalhes podem ser encontrados em [1,2] e na norma ASTM E647 Erro! Fonte de referência não encontrada.. Entretanto, de maneira bastante simplificada, uma amostra é testada sob controle de carga $(P)$ ao longo de milhares de ciclos, com razão de carga $(R=$ $\left.P_{\text {min }} / P_{\text {máx }}\right)$ usualmente superior a zero para minimizar efeitos de fechamento de trincas e tensões residuais [2]. Durante o teste a trinca cresce por fadiga e a amplitude de fator de intensidade de tensões $(\Delta K)$ é incrementada, como ilustra a Figura 2(a). No instante " $i$ " o tamanho de trinca é $a_{i}$, o que significa rigidez $1 / C_{i}$. No momento " $n$ ", $a_{n}>a_{i}$ e a amostra apresenta menor rigidez $1 / C_{n}$ (o que significa maior flexibilidade). Com isto, evoluções da taxa de propagação da trinca com o número de ciclos $(d a / d N)$ podem ser determinadas em função de $\Delta K$ aplicado (ou $\Delta J$ se marcante plasticidade estiver presente $[1,2])$. Tais trajetórias representam a base para previsões de vida de componentes considerando a propagação de trincas à fadiga.

\footnotetext{
* Contribuição técnica ao $69^{\circ}$ Congresso Anual da ABM - Internacional e ao 14ํㅡㄹ ENEMET - Encontro Nacional de Estudantes de Engenharia Metalúrgica, de Materiais e de Minas, 21 a 25 de julho de 2014, São Paulo, SP, Brasil.
} 


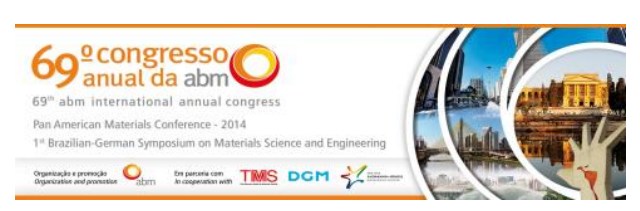

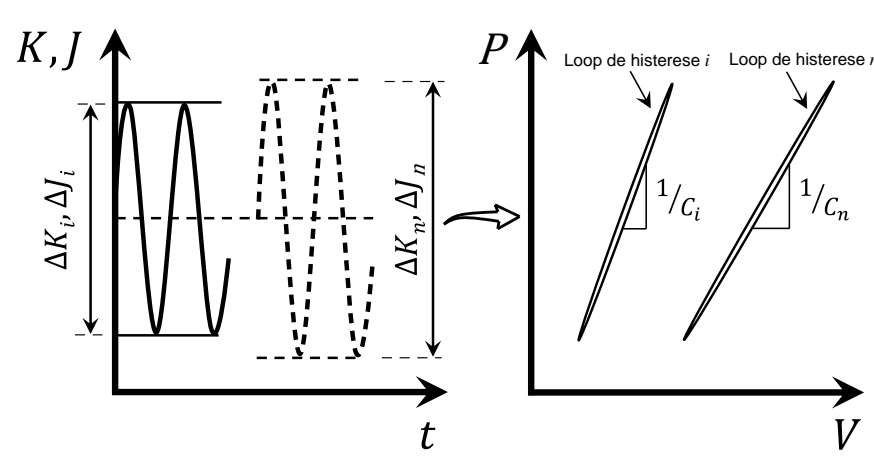

(a)

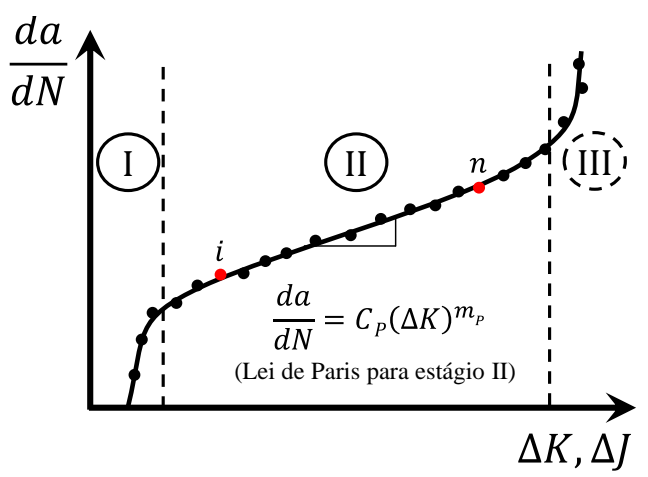

(b)

Figura 2. (a) Carregamento cíclico em controle de carga $(P)$ causando propagação de trinca em fadiga e consequentemente aumento em $\Delta K, \Delta J$ e na flexibilidade $C$. (b) Resultado esquemátivo de curva de propagação de trinca em fadiga incluindo a conhecida Lei de Paris para o estágio II.

\section{MATERIAIS E MÉTODOS}

No presente trabalho, foram utilizadas amostras de um aço estrutural ASTM A36 [15] com composição química apresentada pela Tabela 1. Este material apresenta microestrutura composta de ferrita e perlita, com grãos entre 20 e $30 \mu m$ e muito pouco bandeamento (grãos aproximadamente equiaxiais) mesmo na direção paralela à laminação.

Tabela 1. Composição Química do aço ASTM A36 [15] utilizado no estudo.

\begin{tabular}{ccccccccc}
\hline & $\mathrm{C}$ & $\mathrm{S}$ & $\mathrm{Si}$ & $\mathrm{Cu}$ & $\mathrm{Cr}$ & $\mathrm{Mn}$ & $\mathrm{Ni}$ & $\mathrm{Mo}$ \\
\hline ASTM A36 & 0,13 & 0,014 & 0,2 & 0,008 & 0,017 & 0,76 & 0,012 & 0,003
\end{tabular}

Todas as amostras para ensaios mecânicos foram extraídas da meia espessura de uma chapa laminada a quente de $1 / 2$ polegada, na direção transversal à laminação, a fim de posicionar o entalhe e a pré-trinca de fadiga em um plano paralelo à direção de laminação e que se extende pela espessura do material (Figura 3a). As dimensões básicas das amostras seguem as recomendações da norma para ensaios de impacto Charpy ASTM E23 [16] e um exemplo de amostra em ensaio pode ser visto na Figura 3(b). Entretanto, como ilustra a Figura 4(a), diversos aprimoramentos tiveram de ser implementados nos espécimes, a saber: i) primeiramente, o comprimento usual de $55 \mathrm{~mm}$ foi ampliado para $70 \mathrm{~mm}$, a fim de garantir uma distância entre apoios de $60 \mathrm{~mm}$ na flexão em 3 pontos, dando mais estabilidade ao carregamento; ii) a altura da amostra foi mantida em $10 \mathrm{~mm}$, mas se fez necessária a incorporação de entalhes laterais (side-grooves) de $0,5 \mathrm{~mm}$ em cada lado (1,0 mm no total, ou $10 \%$ da espessura) para favorecer a uniformidade da frente da pré-trinca de fadiga e garantir a propagação da trinca no plano desejado (maiores detalhes são apresentados na seção de resultados); iii) os entalhes convencionais realizados por brochamento em amostras Charpy foram substituídos por entalhes bastante mais elaborados confeccionados por eletro erosão a fio e com características que permitissem a fixação de um extensômetro para a medição do CMOD diretamente na amostra. Duas profundidades de trinca eram objetivadas, sendo uma em torno de $45 \%$ da altura da amostra (usando o entalhe da Figura 4c) e outra em torno de $25 \%$ (entalhe da Figura 4d). Foram confeccionadas 10 amostras com entalhes rasos e $10 \mathrm{com}$ entalhes fundos, sendo metade de cada lote dedicada a ensaios de curva $\mathrm{R}$ e a outra metade a ensaios de propagação por fadiga.

\footnotetext{
* Contribuição técnica ao $69^{\circ}$ Congresso Anual da ABM - Internacional e ao 14ํㅡㄹ ENEMET - Encontro Nacional de Estudantes de Engenharia Metalúrgica, de Materiais e de Minas, 21 a 25 de julho de 2014, São Paulo, SP, Brasil.
} 


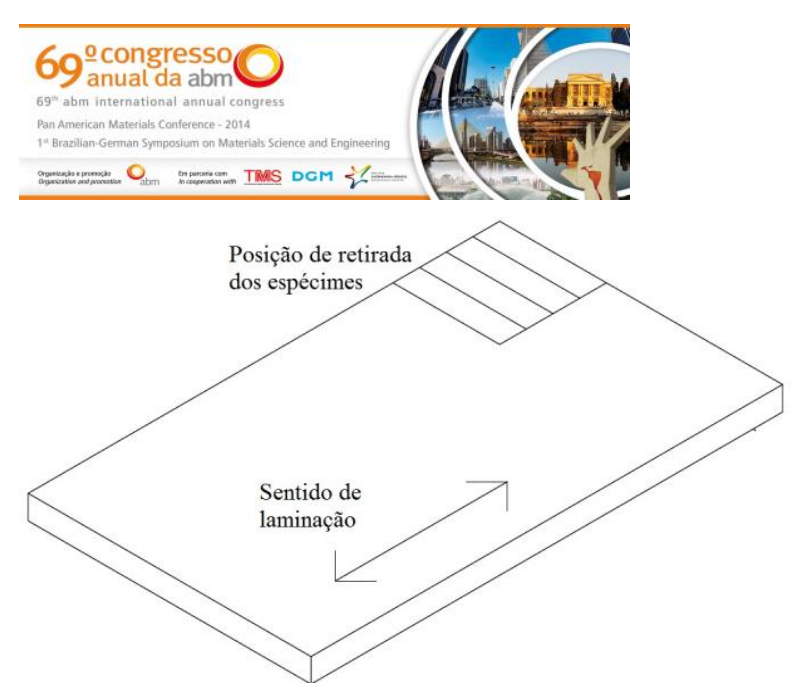

(a)

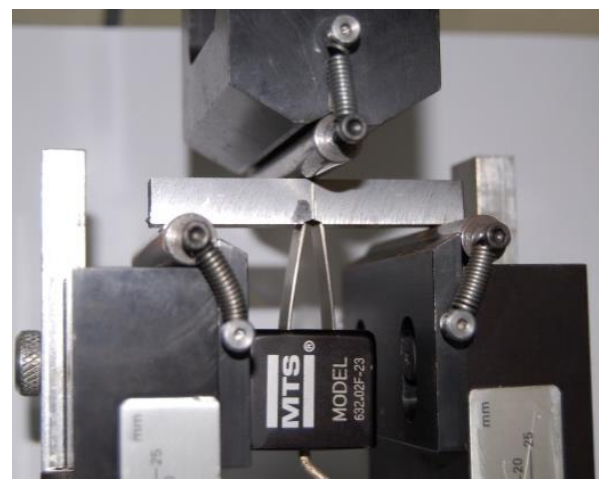

(b)

Figura 3. (a) llustração da orientação de retirada das amostras e (b) amostra com trinca rasa preparada para ensaio na máquina MTS 810 da FEl.

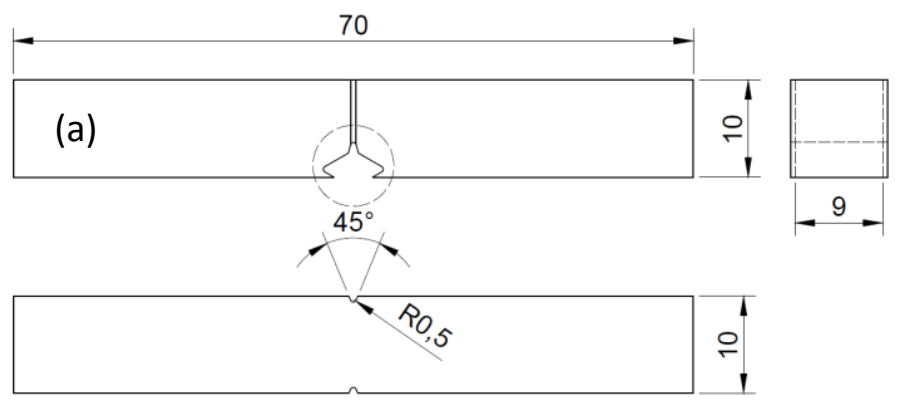

(c)

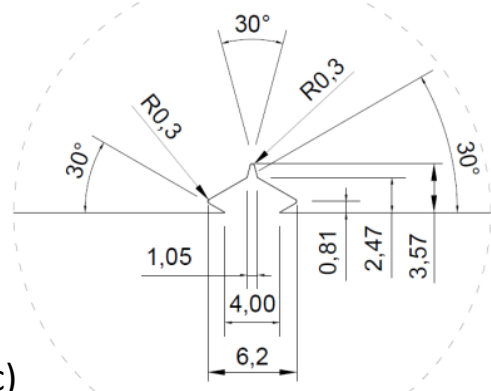

(b)
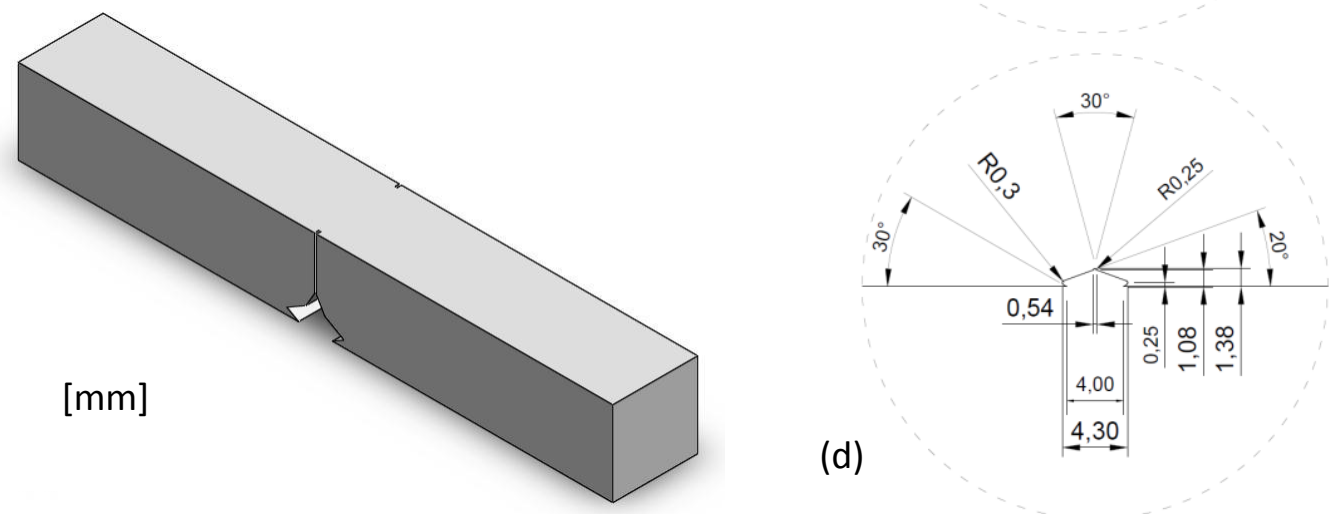

(d)

Figura 4. (a) Dimensões principais das amostras elaboradas, (b) ilustração em perspectiva, (c) detalhe do entalhe aplicável à iniciação de trincas profundas e (d) entalhe para trincas rasas.

Os ensaios foram realizados em máquina universal MTS 810 disponível no Centro de Desenvolvimento de Materiais Metálicos da FEI (CDMatM-FEI) empregando extensômetro MTS 632-02-23. No caso dos ensaios de curva $R$, foi empregada sempre que possível a norma ASTM E1820 [3], velocidade de $0,5 \mathrm{~mm} / \mathrm{min}$ e os descarregamentos parciais foram de $20 \%$. Nos ensaios de propagação por fadiga, foi usada como referência a norma ASTM E647 [5], razão de carga 0,1 e frequência de $10 \mathrm{~Hz}$. Todas as soluções de flexibilidade elástica $(C)$ empregadas para a previsão de comprimento instantâneo de trinca $(a)$ vieram das soluções numéricas do trabalho de Donato e Moreira [14]. Todo o pós-processamento de dados foi realizado por rotinas dedicadas desenvolvidas em linguagem MatLab.

Após concluídos os ensaios, as amostras foram resfriadas em nitrogênio líquido e tiveram seu ligamento remanescente final fraturado. A análise fractográfica foi realizada empregando estereomicroscópio e software dedicado desenvolvido pelo presente grupo de pesquisa para as medições de trinca (Figura 5).

\footnotetext{
* Contribuição técnica ao 69ำ Congresso Anual da ABM - Internacional e ao 14ํㅡㄹ ENEMET - Encontro Nacional de Estudantes de Engenharia Metalúrgica, de Materiais e de Minas, 21 a 25 de julho de 2014, São Paulo, SP, Brasil.
} 


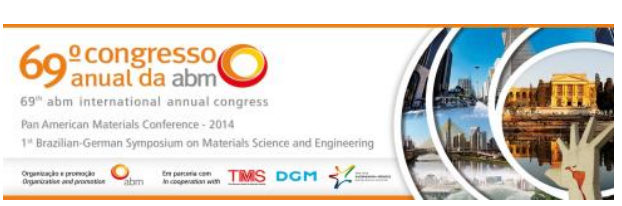

rasgamento dúctil; como esperado, as curvas $R$ para trincas rasas fornecem maiores valores de $J$ que trincas profundas, devido à maior perda de restrição plástica. Entretanto, dentro do interesse do trabalho nos ensaios de curva $R$, os ensaios com amostras PCVN se mostraram plenamente viáveis e controláveis. Trabalhos futuros devem comparar tais curvas com resultados de espécimes $\mathrm{C}(\mathrm{T})$, suportando conclusões sobre dominância $J$ e transferabilidade.

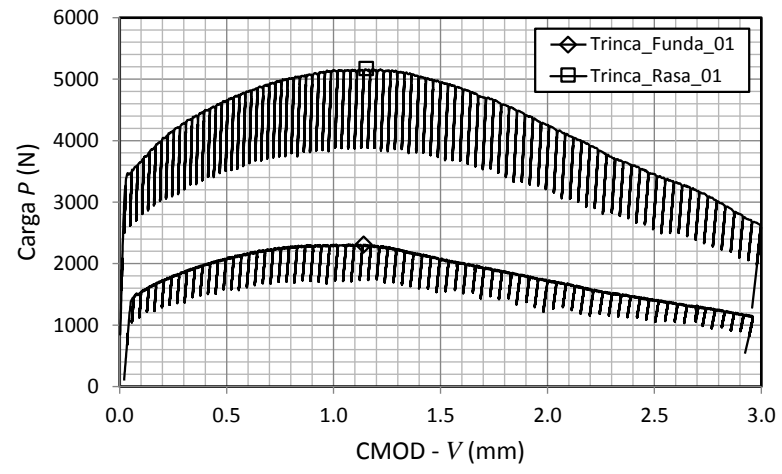

(a)

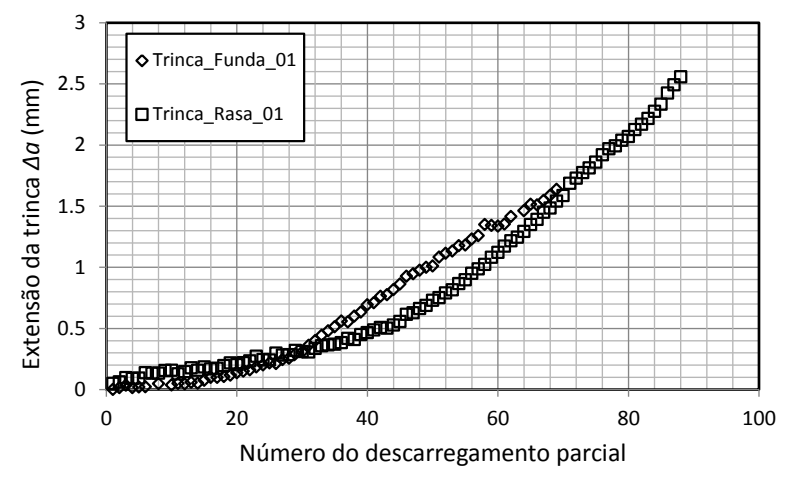

(b)

Figura 8. (a) Curva de carga $(P)$ vs. CMOD $(V)$ obtidas durante ensaios de trincas rasas e profundas e (b) evolução do comprimento de trinca estimado como função dos sucessivos descarregamentos.

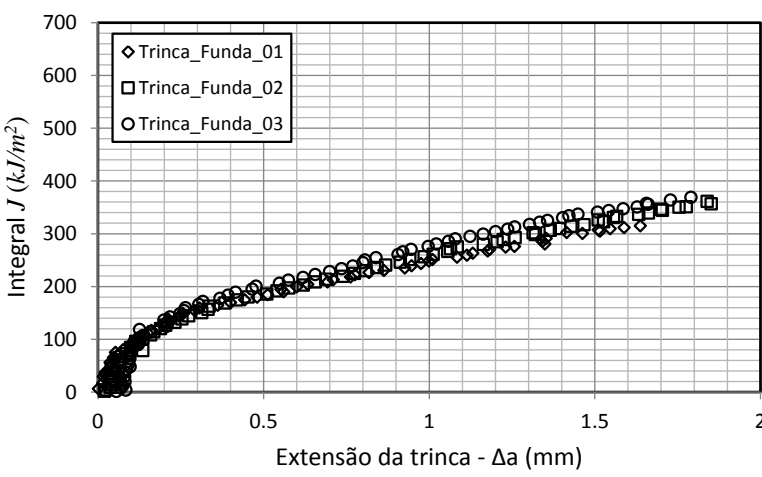

(a)

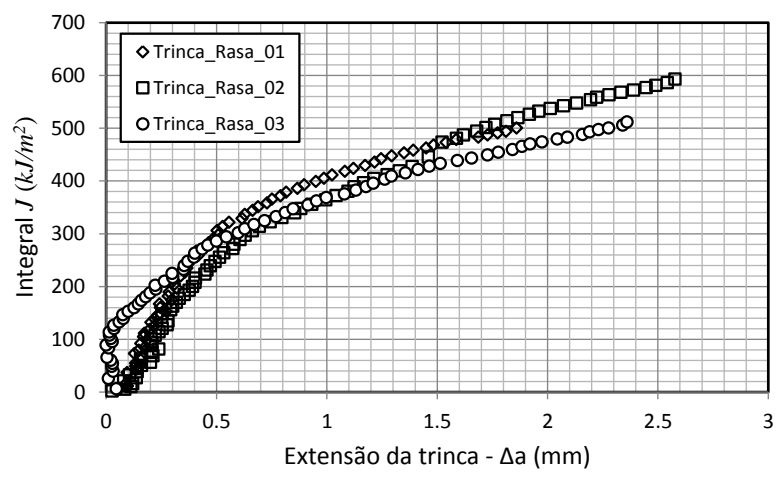

(b)

Figura 9. Curvas $J-R$ obtidas para amostras com (a) trincas profundas e (b) trincas rasas. São evidentes os maiores valores de $J$ para trincas rasas devido à maior perda de restrição plástica.

\subsection{Resultados de curvas $d a / d N$ vs. $\Delta K$}

Analogamente à estabilidade de resultados identificada para os ensaios de curva $R$, a Figura 10(a) apresenta o potencial de acompanhamento do comprimento instantâneo da trinca $(a)$ com a evolução do número de ciclos $(N)$ dos ensaios de fadiga empregando a técnica de flexibilidade elástica. São exemplificados um caso de trinca rasa (se iniciando com $a \sim 2,5 \mathrm{~mm}$ e falhando com $a \sim 6,5 \mathrm{~mm}$ ) e um caso de trinca profunda (se iniciando com $a \sim 4,5 \mathrm{~mm}$ e falhando com $a \sim 7,0 \mathrm{~mm}$ ). Em ambos os casos, chama a atenção o pequeno espalhamento dos resultados de tamanho de trinca instantâneo e também vale notar a falha com aproximadamente mesmo comprimento de trinca final, independente do comprimento inicial (o que era esperado). Especialmente no caso de ensaios $d a / d N v s . \Delta K$, ferramentas acuradas para estimativa do comprimento instantâneo do defeito são fundamentais para o acompanhamento e pós-processamento dos resultados já que em duas amostras das testadas foi ligeiramente complexo distinguir a região de pré-trincamento de fadiga (controlada por $\Delta K$ ) da região de propagação (controlada por carga e consequentemente com $\Delta K$ crescente). Um caso crítico é apresentado na Figura 10(b) (fica evidente a similaridade das regiões) e esta dificuldade é reforçada em

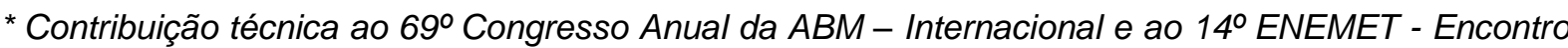
Nacional de Estudantes de Engenharia Metalúrgica, de Materiais e de Minas, 21 a 25 de julho de 2014, São Paulo, SP, Brasil.
} 
amostras de pequenas dimensões pois cargas do final do pré-trincamento e do ensaio podem ser bastante próximas. A comprovação da dimensão medida foi nestes dois casos realizada utilizando microscopia eletrônica de varredura (MEV).

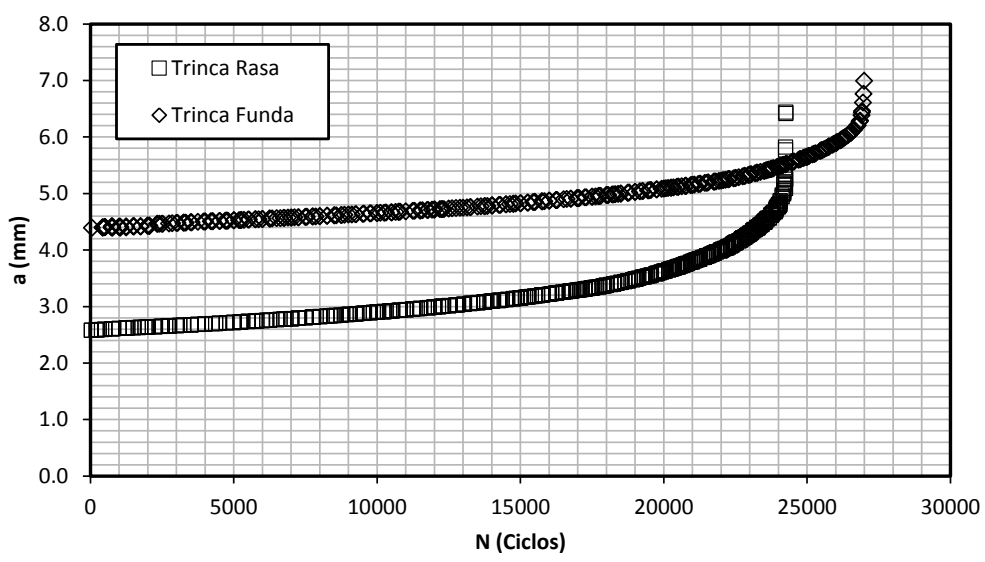

(a)

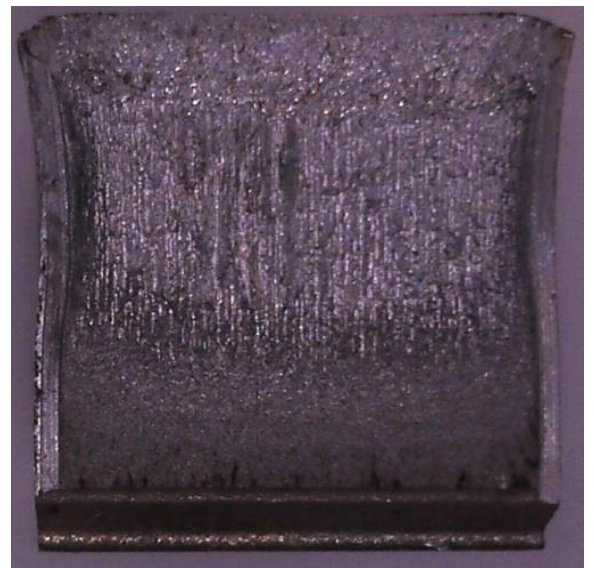

(b)

Figura 10. (a) Comprimento instantâneo das trincas $(a)$ com a evolução do número de ciclos $(N)$ dos ensaios de fadiga empregando a técnica de flexibilidade elástica. (b) Exemplo de amostra na qual as regiões de pré-trinca de fadiga e de propagação em fadiga se confundem em análise macroscópica.

A Figura 11 apresenta exemplos de curvas $d a / d N$ vs. $\Delta K$ obtidas para o aço ASTM A36 em estudo. Uma boa aderência dos pontos experimentais a uma lei de potência é obtida em ambos os casos, especialmente para a região central do estágio II de propagação (vide Figura $2 b$ para referência). Estas figuras preservam todos os dados obtidos dos ensaios, entretanto, os ajustes (já relativamente elevados como pode ser evidenciado pelos $R^{2}$ ) poderiam ser ainda melhorados se pontos nitidamente de instabilidade ou pertencentes aos regimes I e III fossem excluídos. Uma constatação importante em se tratando de boas práticas de ensaio de amostras PCVN é que tais amostras reduzidas apresentaram maior espalhamento dos pontos experimentais próximos à regiões I e III se comparadas a amostras $C(T)$ ou $S E(B)$.

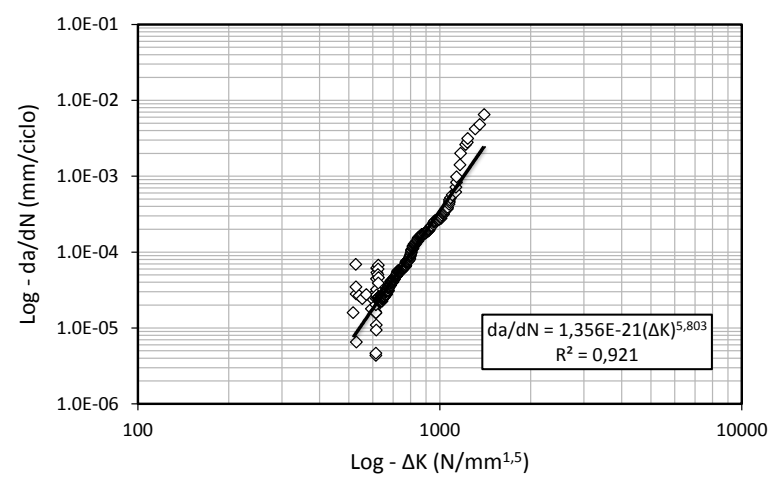

(a - trinca funda)

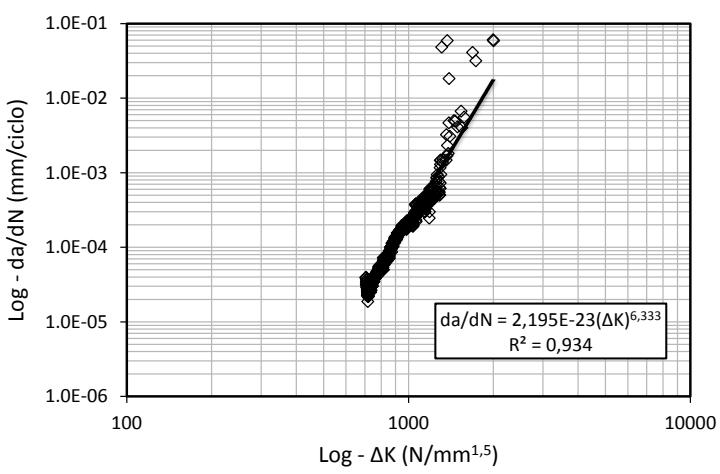

(b - trinca rasa)

Figura 11. Evoluções $d a / d N$ vs. $\Delta K$ ilustrativas dos resultados obtidos para amostras contendo (a) trincas fundas e $(b)$ trincas rasas.

Com base em curvas $d a / d N$ vs. $\Delta K$ como as ilustradas na Figura 11, a Figura 12 apresenta os ajustes por lei de potência (Lei de Paris) para todas as amostras PCVN ensaiadas à fadiga. Tanto resultados para trincas rasas como trincas profundas estão apresentados e fica evidente a proximidade entre as evoluções encontradas. A Tabela 3 complementa tais resultados com os valores médios de $C_{P}$ e $m_{P}$ do modelo de Paris acompanhados dos coeficientes de múltipla correlação $\left(R^{2}\right)$ médios. Vale notar a boa aderência dos dados experimentais ao modelo de potência adotado,

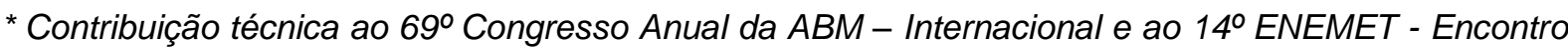
Nacional de Estudantes de Engenharia Metalúrgica, de Materiais e de Minas, 21 a 25 de julho de 2014, São Paulo, SP, Brasil.
} 


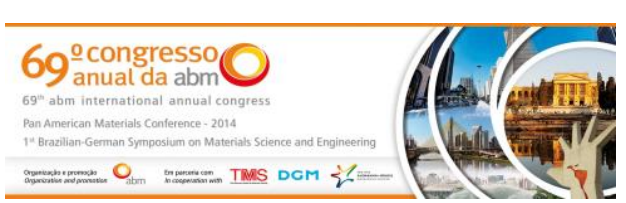

além da proximidade de propriedades mecânicas obtidas com as amostras PCVN, em especial $m_{P}$, parâmetro de grande relevância pois caracteriza a progressividade da taxa de crescimento do defeito com a força motriz de trinca $(\Delta K)$ aplicada. Assim como nos ensaios de curva $R$, tais resultados encorajam o uso de amostras PCVN para ensaios $d a / d N$ vs. $\Delta K$, desde que futuras considerações de dominância e transferabilidade sejam endereçadas.

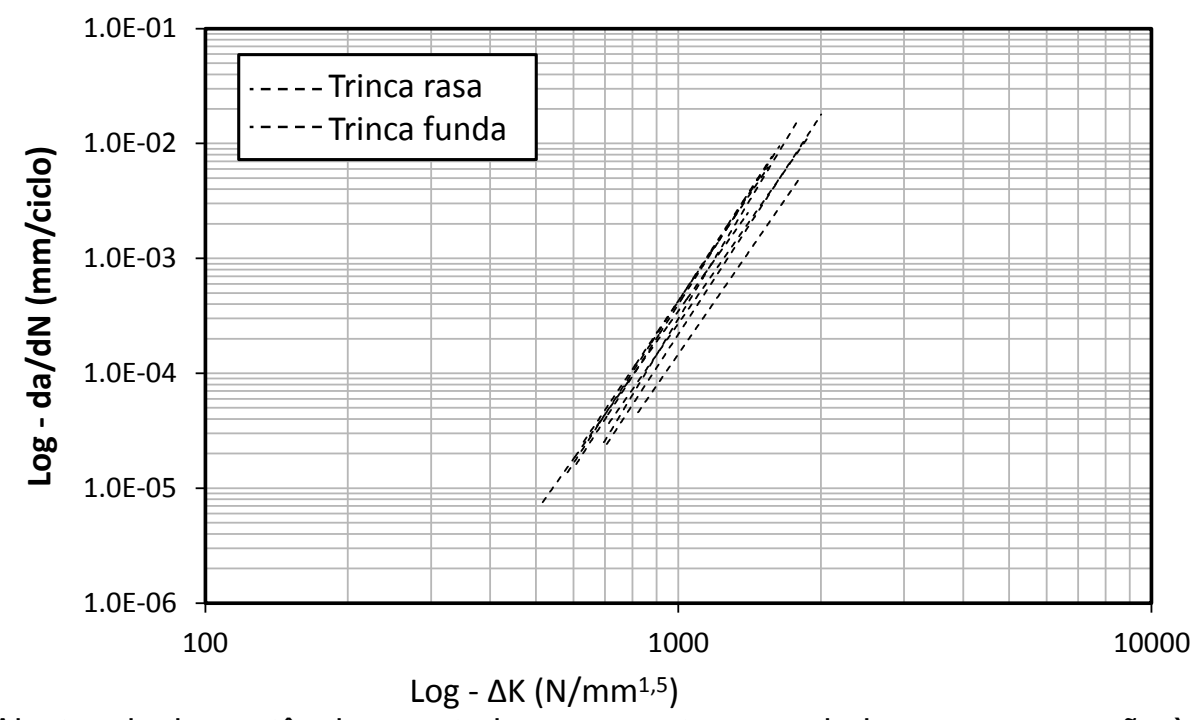

Figura 12. Ajustes do tipo potência para todas as amostras ensaiadas em propagação à fadiga, tanto considerando trincas rasas como profundas..

Tabela 3. Propriedades de resistência à propagação de trincas por fadiga do aço ASTM A36.

\begin{tabular}{cccc}
\hline ASTM A36 & $C_{P}$ & $m_{P}$ & $R^{2}$ \\
\hline Trinca rasa & $1,770 \mathrm{E}-22$ & 6,358 & 0,92 \\
\hline Trinca funda & $3.956 \mathrm{E}-22$ & 6,186 & 0,94 \\
\hline
\end{tabular}

\section{CONCLUSÕES}

De posse dos resultados obtidos, algumas conclusões centrais emergem:

- Embora diversos pesquisadores tenham reportado dificuldades na adaptação das geometrias PCVN para ensaios de fratura (curva $R$ ) e fadiga ( $d a / d N$ vs. $\Delta K$ ) e a obtenção de resultados com expressivo espalhamento experimental, os resultados obtidos nesta investigação apontam para a viabilidade de realização de tais experimentos com repetibilidade e adequado controle de $P$ e $V$.

- Os entalhes projetados e confeccionados por eletro-erosão a fio, juntamente com o método de flexibilidade elástica, garantiram boa transdução do CMOD e coerência das previsões de tamanho de trinca instantânea com as observações da análise post-mortem.

- A incorporação de entalhes laterais com $10 \%$ da espessura se mostrou fundamental para a garantia da estabilidade da frente da pré-trinca, assim como da trinca em extensão/propagação.

- As curvas $J-R$ obtidas apresentaram o comportamento crescente esperado e excelente coincidência entre amostras de mesma geometria. No entanto,

\footnotetext{
* Contribuição técnica ao 69ำ Congresso Anual da ABM - Internacional e ao 14ํㅡㄹ ENEMET - Encontro Nacional de Estudantes de Engenharia Metalúrgica, de Materiais e de Minas, 21 a 25 de julho de 2014, São Paulo, SP, Brasil.
} 
amostras com trincas rasas apresentaram maiores valores de $J$ como resultado da maior perda de restrição plástica, o que era esperado.

- As evoluções $d a / d N$ vs. $\Delta K$ apresentaram elevada proximidade entre todas as amostras avaliadas, tanto com trincas rasas como profundas, em parte devido à menor incidência de plasticidade no controle do micromecanismo de dano.

- No geral, os resultados obtidos sinalizam a viabilidade de ensaio de amostras PCVN para os ensaios de interesse do trabalho e motivam a continuidade dos estudos. Tal sequência inclui incorporar comparações dos resultados com amostras compactas $\mathrm{C}(\mathrm{T})$ de alta restrição plástica e também avaliações de dominância $K$ e $J$ a fim de discutir em profundidade questões de transferabilidade.

\section{Agradecimentos}

Este trabalho é suportado pelo Centro Universitário da FEI por meio de recursos materiais, humanos e infra-estrutura laboratorial. Adicionalmente, os autores gostariam de agradecer ao CNPQ pelas bolsas PIBIC concedidas a RFM e TRM e à CAPES pela bolsa de mestrado concedida ao aluno FCM.

\section{REFERÊNCIAS}

1 Anderson TL. Fracture Mechanics: Fundamentals and Applications- $3^{\text {rd }}$ edition, CRC Press, New York, 2005.

2 Suresh S. Fatigue of Materials. 2nd edition, Cambridge University Press, United Kingdom, 1998.

3 American Society for Testing and Materials (ASTM), 2011, "Standard Test Method for Measurement of Fracture Toughness.", ASTM E 1820, Philadelphia, 2011.

4 International Organization for Standardization (ISO). Metallic Materials - Unified method of test for the determination of quasistatic fracture toughness - ISO 12135, ISO International, 2002.

5 American Society for Testing and Materials (ASTM), 2013, "Standard Test Method for Measurement of Fatigue Crack Growth Rates.", ASTM E647, Philadelphia, 2013.

6 American Petroleum Institute - API. Specification for pipeline, API specification 5L. 44ed., 2007.

7 Koppenhoefer KC, Dodds JrRH. Ductile crack growth in pre-cracked CVN specimens: numerical studies, Nuclear Engineering and Design, 1989; 180: 221-241.

8 Koppenhoefer KC, Dodds JrRH. A Numerical Investigation of Loading Rate Effects on Pre-Cracked Charpy V-Notch Specimens. Technical Report of the University of Illinois at Urbana-Champaign, 1997.

9 Chaouadi R, Puzzolante JL. Loading rate effect on ductile crack resistance of steels using precracked Charpy specimens. International Journal of Pressure Vessels and Piping, 2008; 85(11): 752-761.

10 Puigh RJ, Bauer RE, Ermi AM, Chin BA. Miniaturized fatigue crack growth specimen technology and results. Journal of Nuclear Materials, 1981; 104: 1501-1504.

11 Sriharsha HK, Pandey RK, Chatterjee S. Towards standardizing a sub-size specimen for fatigue crack propagation behaviour or a nuclear pressure vessel steel, Engineering Fracture Mechanics, 1999; 64: 607-624.

12 Li M, Stubbins JF. Subsize specimens for fatigue crack growth rate testing of metallic materials. In: Sokolov MA, Landes JD, Lucas GE, editors. "Small specimen test techniques: fourth volume", ASTM STP 1418. West Conshohocken (PA): ASTM International, 2002; 321-335.

13 Shin CS, Lin SW. Evaluating fatigue crack propagation properties using miniature specimens", International Journal of Fatigue, 2012; 43: 105-110.

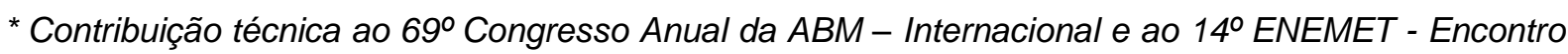
Nacional de Estudantes de Engenharia Metalúrgica, de Materiais e de Minas, 21 a 25 de julho de 2014, São Paulo, SP, Brasil.
} 
14 Donato GHB, Moreira FC. Effects of side-grooves and 3-D geometries on compliance solutions and crack size estimations applicable to $C(T)$, SE(B) and clamped SE(T) specimens, In: Proc. of the ASME Pressure Vessels and Piping Division Conference (PVP 2013), Paris, França, 2013.

15 American Society for Testing and Materials (ASTM), Standard Specification for Carbon Structural Steel., ASTM A36, Philadelphia, 2012.

16 American Society for Testing and Materials (ASTM), Standard Test Methods for Notched Bar Impact Testing of Metallic Materials., ASTM E23, Philadelphia, 2012.

17 Bao R, Yang H, Zhang J., Peng L, Fei B. Fatigue crack growth measurement in a superalloy at elevated temperature. International Journal of Fatigue, 2013; 47.

18 American Society for Testing and Materials (ASTM), Standard Test Methods for Tension Testing of Metallic Materials. ASTM E8/E8M, Philadelphia, 2013.

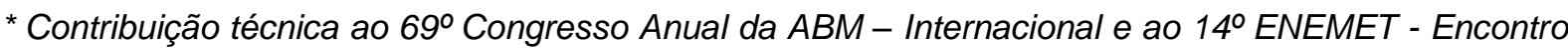
Nacional de Estudantes de Engenharia Metalúrgica, de Materiais e de Minas, 21 a 25 de julho de 2014, São Paulo, SP, Brasil. 